\title{
Fatores Prognósticos de Mortalidade por Covid-19 no Rio Grande do Norte, Brasil
}

\author{
Mortality Prognostic Factors for Covid-19 in Rio Grande do Norte, Brazil
}

\section{RESUMO}

Objetivo: Avaliar a associação das comorbidades e dos sintomas com os óbitos entre os casos confirmados de Covid-19 no Estado do Rio Grande do Norte, Brasil. Metodologia: Trata-se de um estudo transversal, onde foram selecionados 44.098 casos confirmados, oficialmente divulgados, dos quais 1.615 foram óbitos, cujas variáveis preditoras foram: idoso, branco, diabetes, doenças cardíacas, respiratórias e renais, imunossupressão, tosse, febre, dor de garganta, e dispneia. Modelos regressivos clássicos e bayesiano de Poisson foram usados para ajuste das razões de taxa de incidência de mortalidade. Resultados: As mortes corresponderam a 3,7\% dos casos confirmados de Covid-19. Os idosos com Covid-19 tiveram 10 (ICr95\%: 8,25$12,74)$ vezes mais chances de óbito que a sua contraparte. 0 excedente de mortes foi $34 \%$ (ICr95\%: 1,12-1,60) maior entre os homens, contudo, a raça/cor branca foi fator de proteção (RTI: 0,63; ICr95\%: 0,50-0,79). As comorbidades, como o diabetes (RTI: 2,13), as doenças cardíacas (RTI: 2,03 ), as respiratórias (RTI: 1,90$)$ e as imunossupressoras (RTI: 2,89$)$, além de sintomas como a tosse $(\mathrm{RTI}: 1,39)$ e a dispneia $(\mathrm{RTI}: 1,52)$ foram fatores prognósticos associados à mortalidade por Covid-19. Conclusão: Os idosos apresentaram excedente de mortalidade por Covid-19 no Estado do Rio Grande do Norte, como também os homens e as pessoas que apresentaram comorbidades como o diabetes, as doenças cardíacas, as doenças respiratórias e as imunossupressoras, além de sintomas como a tosse e a dispneia, estiveram associados à maior mortalidade.

\section{DESCRITORES}

Infecções por Coronavírus. Óbito. Pandemia. Fatores de Risco.

\begin{abstract}
Objective: the study was to evaluate the association of comorbidities and symptoms with the deaths among confirmed cases of covid-19 in the state of Rio Grande do Norte, Brazil. Methodology: This was a cross-sectional study, in which 44.098 confirmed cases, officially disclosed, were selected, of which 1.615 were deaths, whose predictor variables were: elderly, white, diabetes, heart, respiratory and renal diseases, immunosuppression, cough, fever, sore throat, and dyspnea. Bayesian and Classical Analysis of Poisson Regression were used to adjust the mortality incidence rate ratios. Results: Deaths corresponded to $3.7 \%$ of confirmed cases of covid-19. Elderly people with covid-19 were 10 (95\% Cl: 8.25-12.74) times more likely to die than their counterpart. The surplus of deaths was $34 \%(95 \% \mathrm{Cl}: 1.12-1.60)$ higher among men, but the white race/ color was a protective factor (IRR: $0.63 ; 95 \% \mathrm{Crl}: 0.50-0.79$ ). Comorbidities such as diabetes (IRR: 2.13), heart (IRR: 2.03), respiratory (IRR: 1.90) and immunosuppressive (IRR: 2.89) diseases, in addition to symptoms such as coughing (IRR: 1.39) and dyspnea (IRR: 1.52) were prognostic factors associated with covid-19 mortality. Conclusion: The elderly people had a surplus of mortality due to covid-19 in Rio Grande do Norte. Also men and people who presented comorbidities such as diabetes, heart diseases, respiratory diseases and immunosuppression, in addition to symptoms such as cough and dyspnea, were associated with higher mortality.
\end{abstract}

\section{DESCRIPTORS}

Coronavirus Infections. Death. Pandemics. Risk Factors.

\footnotetext{
${ }^{1}$ Médico veterinário, do Departamento de Vigilância em Saúde, Prefeitura Municipal de Natal, Brasil.

${ }_{2}$ Professor Dr. no Programa de Pós-graduação em Saúde Coletiva, Departamento de Odontologia, Universidade Federal do Rio Grande do Norte, Natal-RN.
} 
A covid-19 é uma doença causada por um novo betacoronavírus RNA envelopado conhecido como coronavírus da síndrome respiratória aguda grave (SARS-CoV-2) e que apresenta diversos sintomas, especialmente febre, tosse, dor de garganta e dispneia ${ }^{1}$. A transmissão por via aérea é rápida e representa a principal rota de transmissão para disseminação da doença. Portanto, o uso de máscaras faciais de proteção, o distanciamento e isolamento social, além das medidas de higiene são armas fundamentais no combate à doença ${ }^{2}$.

A Organização Mundial de Saúde enfatizou que uma das principais questões a serem abordadas durante a pandemia de covid-19 é entender os fatores que interferem na severidade da doença ${ }^{3}$. Várias comorbidades, incluindo hipertensão, doença cardiovascular, diabetes, doença cerebrovascular maligna, doença pulmonar obstrutiva crônica, e nefropatia crônica podem estar envolvidas na severidade da covid-19 e evolução para óbito, especialmente as três primeiras condições ${ }^{4}$. Além disso, a idade mais avançada, especialmente por estar relacionada a uma maior carga de comorbidades, é considerada um fator de risco importante na covid- $19^{5}$. Nestes aspectos, a pandemia impacta a vida das pessoas, especialmente entre os idosos, que precisam manter-se em isolamento social por muito tempo, comprometendo a atividade física para condicionamento cardiovascular, de força e massa muscular, além da saúde mental ${ }^{6}$.

Em 31 de dezembro de 2019, foram detectados os primeiros casos de covid-19 na cidade de Wuhan, na província de Hubei, Chi$\mathrm{na}^{7}$, apesar desse vírus já circular na Europa desde 12 de março daquele $a^{\circ}{ }^{8}$. O primeiro caso de covid-19 no Brasil foi confirmado em 26 de fevereiro de 2020, em São Paulo-SP. Após 21 dias, foi registrado o primeiro óbito pela doença no país ${ }^{9}$. Em 31 de julho, o Brasil se encontrava na segunda posição em incidência (2,6 milhões de casos confirmados) e de mortalidade (91,3 mil óbitos) no mundo ${ }^{10}$. Assim, o Brasil se tornou epicentro da pandemia de covid-19 na América Latina ${ }^{11}$.

A distribuição dos óbitos entre as unidades federativas brasileiras é altamente heterogênea, com cinco estados - São Paulo, Rio de Janeiro, Ceará, Pernambuco e Amazonas - contando com $81 \%$ dos óbitos, até 08 de maio de $2020^{11}$. Neste cenário, o Rio Grande do Norte com 1,7 mil óbitos e 48,8 mil casos confirmados, apresentava 22,9 mil recuperados e 736 casos ativos $^{12}$. Apesar do Rio Grande do Norte apresentar a segunda melhor distribuição na relação entre leitos de UTI e população residente no Nordeste, as taxas de ocupação chegaram a 100\% com muitas pessoas à espera de atendimento ${ }^{13}$.

Em busca de contribuir com infor- 
mações relevantes sobre a mortalidade por covid-19 no Rio Grande do Norte e servir como parâmetro clínico-epidemiológico da mortalidade para fins comparativos, este estudo buscou avaliar a associação das comorbidades e sintomas com os óbitos entre pessoas confirmadas com covid-19.

\section{METODOLOGIA}

Este é um estudo transversal, baseado nos dados disponibilizados pelo boletim epidemiológico $\mathrm{n}^{\circ} 118$, da Secretaria de Estado da Saúde Pública (SESAP), no Rio Grande do Norte. A base de dados dispõe de 191.892 observações. No total, após depuração das informações, foram selecionados 44.098 casos confirmados, dos quais 1.615 foram óbitos $(3,66 \%)$. Foram 169 casos confirmados e seis óbitos a menos que as informações divulgadas naquele boletim.

A variável dependente do estudo foram os óbitos por covid-19. As variáveis independentes do tipo dummy foram obtidas das informações disponíveis para cada indivíduo com relação às características individuais, comorbidades e sintomas clássicos da covid-19, quais sejam: idoso, branco, diabetes, doenças cardíacas, doenças respiratórias, doenças renais, imunossupressão, tosse, febre, dor de garganta, e dispneia.

Foram procedidas análises de regres- são de Poisson, segundo o sexo dos indivíduos, considerando-se o modelo frequentista. No modelo de regressão de Poisson, a taxa de incidência de mortalidade para a observação $j$ foi assumida como: $r_{j}=e^{\beta 0+\beta_{1 \times 1 j}}+\ldots+\beta_{1 \times 1 j}(1.1)$. A comparação das taxas foi feita através de razões de taxa de incidência (RTI). Todas as variáveis independentes estatisticamente significantes para ambos sexos se mantiveram para a análise sem estratificação, desde que apresentassem homogeneidade avaliada através do método de Mantel-Haenszel (M-H), usando um nível de significância de $\alpha=0,05$.

A regressão bayesiana de Poisson foi conduzida com o modelo final descrito anteriormente. Foram consideradas 165.000 iterações pelo método de Monte Carlo via cadeias de Markov (MCMC) e tamanho da amostra MCMC de 140.000; um período de aquecimento (burn-in), que representa o número de iterações excluídas para minimizar efeitos inicial, igual a 25.000; e o thinning igual a um, isto é, todos os valores simulados foram salvos. O diagnóstico da convergência de Gelman-Rubin, $R_{c}$, foi utilizado para avaliar a convergência MCMC pela análise das diferenças entre quatro cadeias múltiplas de Markov. Assim, valores de Rc inferiores a 1,1 para todos os parâmetros do modelo final indicam convergência. Todas as análises estatísticas foram conduzidas com o pacote estatístico Stata/MP 16.0. 
Considerando-se que os dados usados neste estudo são anônimos e a disponibilidade é de domínio público, a submissão a Comissão de Ética não foi necessária, em conformidade com as recomendações da Comissão Nacional de Ética em Pesquisa (Conep).

\section{RESULTADOS}

Em $11^{\circ}$ lugar na posição da taxa de mortalidade por covid-19 no país $(46,1$ óbitos/100 mil habitantes), as mortes corresponderam a $3,7 \%$ dos casos confirmados no Rio Grande do Norte (Tabela 1). Os homens apresentaram um excesso de óbitos maior (38\%), inclusive quando comparados em cada fator avaliado neste estudo.
A média de idade dos óbitos foi de 67 anos, sendo 69 entre as mulheres e 67 anos entre os homens. Portanto, os idosos foram o grupo etário de maior risco de morte por covid-19 (Tabela 2). Além disso, as desigualdades sociais foram captadas pela menor mortalidade entre os brancos $(R T I=0,63$; $p$-valor<0,001), independentemente do sexo.

Dentre os casos confirmados de covid-19, 18,7\% (IC95\%: 18,32-19,05) apresentavam pelo menos uma das cinco comorbidades avaliadas, sendo $11,3 \%$ de óbitos neste grupo e $1,9 \%$ naqueles sem comorbidades. A imunossupressão, diabetes, doenças cardíacas e respiratórias foram associados a maior mortalidade, o que não ocorreu com as doenças renais (Tabela 2 e 3 ).

Tabela 1. Incidência de mortalidade entre pessoas com covid-19, no Rio Grande do Norte, Brasil

\begin{tabular}{lcccccccc}
\multirow{2}{*}{ Variáveis } & \multicolumn{2}{c}{ Homens } & \multicolumn{2}{c}{ Mulheres } & & Razão & \multicolumn{2}{c}{ Total } \\
& $\mathrm{n}$ & $\%$ & $\mathrm{n}$ & $\%$ & & $(\mathrm{M} / \mathrm{H})$ & $\mathrm{n}$ & $\%$ \\
\hline Idoso & 621 & 16,63 & 533 & 12,47 & 1,33 & 1154 & 14,41 \\
Branco & 161 & 3,00 & 136 & 1,97 & 1,52 & 297 & 2,42 \\
Diabetes & 285 & 19,17 & 257 & 13,41 & 1,43 & 542 & 15,92 \\
D. cardíacas & 367 & 15,21 & 302 & 10,89 & 1,40 & 669 & 12,90 \\
D. respiratórias & 47 & 9,71 & 45 & 6,27 & 1,55 & 92 & 7,65 \\
D. renais & 43 & 23,50 & 24 & 13,64 & 1,72 & 67 & 18,66 \\
Imunossupressão & 09 & 8,04 & 14 & 7,18 & 1,12 & 23 & 7,49 \\
Tosse & 483 & 5,01 & 385 & 3,61 & 1,39 & 868 & 4,28 \\
Febre & 443 & 4,94 & 329 & 3,67 & 1,35 & 772 & 4,31 \\
Dor de garganta & 225 & 3,93 & 186 & 2,56 & 1,53 & 411 & 3,16 \\
Dispneia & 270 & 7,66 & 183 & 4,67 & 1,64 & 453 & 6,08 \\
\hline Total & 896 & 4,29 & 719 & 3,10 & 1,38 & 1615 & 3,66 \\
\hline
\end{tabular}

*Desde 10 de fevereiro (primeiro óbito) a 20 de julho de 2020. 
Tabela 2. Regressão clássica de Poisson, com razão de taxas de incidência de mortalidade por covid-19 e testes de homogeneidade de Mantel-Haenszel, no Rio Grande do Norte, Brasil (2020)

\begin{tabular}{lccccccc}
\hline \multirow{2}{*}{ Variáveis } & \multicolumn{2}{c}{ Mulheres } & \multicolumn{2}{c}{ Homens } & M-H** & \multicolumn{2}{c}{ Todos } \\
\cline { 2 - 8 } & RTI $(E P)^{*}$ & $p$-valor & RTI $(E P)$ & $p$-valor & $p$-valor & RTI $(E P)$ & $p$-valor \\
\hline Homem & - & - & - & - & - & $1,33(1,12)$ & 0,002 \\
Idoso & $10,11(1,60)$ & $<0,001$ & $11,05(1,82)$ & $<0,001$ & 0,843 & $10,21(1,13)$ & $<0,001$ \\
Branco & $0,66(0,11)$ & 0,013 & $0,60(0,10)$ & 0,002 & 0,279 & $0,63(0,07)$ & $<0,001$ \\
Diabetes & $2,49(0,38)$ & $<0,001$ & $1,94(0,31)$ & $<0,001$ & 0,240 & $2,12(0,23)$ & $<0,001$ \\
D. cardíacas & $2,08(0,31)$ & $<0,001$ & $1,98(0,30)$ & $<0,001$ & 0,117 & $2,02(0,21)$ & $<0,001$ \\
D. respiratórias & $1,85(0,51)$ & 0,026 & $1,86(0,46)$ & 0,013 & 0,350 & $1,93(0,35)$ & $<0,001$ \\
D. renais & $1,75(0,69)$ & 0,153 & $1,06(0,48)$ & 0,904 & 0,171 & - & - \\
Imunossupres- & $1,34(0,79)$ & 0,620 & $3,26(1,02)$ & $<0,001$ & 0,786 & & \\
são & & & & & & $2,87(0,74)$ & $<0,001$ \\
Tosse & $1,45(0,21)$ & 0,012 & $1,28(0,19)$ & 0,090 & 0,843 & $1,38(0,14)$ & 0,001 \\
Febre & $0,99(0,14)$ & 0,949 & $1,28(0,18)$ & 0,085 & 0,591 & - & - \\
Dor de garganta & $0,86(0,13)$ & 0,312 & $0,76(0,11)$ & 0,064 & 0,158 & - & - \\
Dispneia & $1,22(0,19)$ & 0,190 & $1,56(0,23)$ & 0,003 & 0,054 & $1,50(0,15)$ & $<0,001$ \\
\multicolumn{1}{c}{ Intercepto: } & $0,00(0,00)$ & $<0,001$ & $0,00(0,00)$ & $<0,001$ & - & $0,00(0,00)$ & $<0,001$ \\
\hline
\end{tabular}

*RTI - Razão de taxa de incidência; EP - Erro padrão. ** Teste de homogeneidade pelo método de Mantel-Haenszel, em nível de significância de $\alpha=0,05$.

Houve 95\% de chance do excedente de mortes ser, em média, três vezes maior entre as pessoas imunossuprimidas confirmadas para covid-19, quando comparadas as suas contrapartes e controladas pelas demais variáveis. Outrossim, a tosse e a dispneia foram os sintomas que apresentaram maiores e significativos excedentes de mortes em pacientes com covid-19. No entanto, quando estratificado por sexo, a tosse foi um fator de risco significativo entre as mulheres $(R T I=1,45$; $p$-valor $=0,012$ ), porém não entendida como confundimento, conforme demonstrado pelo teste de homogeneidade.

\section{DISCUSSÃO}

A diferença de gênero na mortalidade da covid-19 foi encontrada no Brasil e no mundo, sendo explicada por diversos fatores, entre os quais estão os aspectos socioculturais de gênero (maior probabilidade de fumar ou menor higienização das mãos entre os homens) e a susceptibilidade biológica (hor- 
Tabela 3. Regressão bayesiana de Poisson, com razão de taxas de incidência de mortalidade por covid-19, no Rio Grande do Norte, Brasil

\begin{tabular}{lcccccc}
\hline \multirow{2}{*}{ Variáveis } & RTI (MCSE) & \multirow{2}{*}{ Mediana } & \multicolumn{2}{c}{ Intervalo Credível 95\% } & \multirow{2}{*}{ ESS** } & \multirow{2}{*}{$\mathrm{R}_{\mathrm{c}}^{* * *}$} \\
\cline { 1 - 3 } Homem & $1,34(0,004)$ & 1,34 & 1,12 & 1,60 & 1112,58 & 1,01 \\
Idoso & $10,30(0,036)$ & 10,2 & 8,25 & 12,74 & 988,84 & 1,00 \\
Branco & $0,63(0,002)$ & 0,63 & 0,50 & 0,79 & 1376,54 & 1,00 \\
Diabetes & $2,13(0,007)$ & 2,12 & 1,71 & 2,64 & 1073,81 & 1,01 \\
D. cardíacas & $2,03(0,012)$ & 2,02 & 1,65 & 2,50 & 338,18 & 1,01 \\
D. respiratórias & $1,90(0,014)$ & 1,87 & 1,28 & 2,65 & 626,55 & 1,01 \\
Imunossupressão & $2,89(0,028)$ & 2,82 & 1,66 & 4,57 & 694,73 & 1,01 \\
Tosse & $1,39(0,002)$ & 1,38 & 1,14 & 1,68 & 4242,18 & 1,00 \\
Dispneia & $1,52(0,007)$ & 1,51 & 1,24 & 1,84 & 483,65 & 1,00 \\
\multicolumn{1}{c}{ Intercepto: } & $0,003(<0,001)$ & 0,003 & 0,002 & 0,003 & 1033,79 & 1,00 \\
\hline
\end{tabular}

Regressão bayesiana de Poisson: convergência alcançada com 165 mil interações MCMC; tamanho da amostra de MCMC

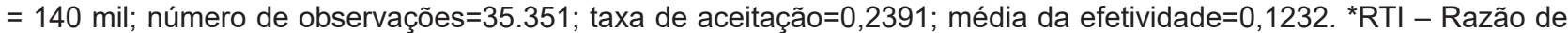
taxa de incidência; **ESS - efective sample size (tamanho efetivo da amostra); ${ }^{* * *}$ Diagnóstico de convergência de Gelman-Rubin (Rc<1,1 indica convergência).

mônios relacionados ao sexo, resposta imune e a concentração de enzimas conversoras da angiotensina 2 - ECA2) ${ }^{14}$.

Foi demonstrado que as porcentagens de casos fatais aumentam com a idade avançada, de nenhuma morte relatada entre pessoas com idade inferior a 19 anos e de $10-27 \%$ em idosos com idade igual ou superior a 85 anos $^{15}$. Além disso, em função do isolamento social, eles apresentam maior risco de desenvolver doenças cardiovasculares, deterioração cognitiva, depressão, ausência de vitamina $D$, solidão, aumento de massa corpórea e polifarmácia ${ }^{6}$. Tudo isso em meio a um contexto de amplas desigualdades sociais em saúde, comprometendo a proteção social deste grupo ${ }^{16}$.

Também foi reportado que negros, asiáticos e outras minorias étnicas apresentam maior risco de adquirir infecção por SARS-CoV-2, mas também piores condições clínicas da covid-1917. Portanto, o risco de evolução para óbito nestes grupos é maior ${ }^{18}$. Um estudo estadunidense apontou que adultos negros não hispânicos de meia idade começam com um nível mais alto de carga de doenças crônicas e desenvolvem multimorbidade em idade mais precoce, em média, que os 
brancos não hispânicos ${ }^{19}$, podendo explicar, ao menos em parte, as condições mais graves de covid-19. Entretanto, a raça negra não foi associada com as elevadas mortalidades hospitalares após ajuste pelas diferenças nas características sociodemográficas e características clínicas na admissão hospitalar ${ }^{20}$.

As comorbidades são fatores importantes que podem determinar maior gravidade da covid-19 e consequente evolução para óbito, especialmente a obesidade, doenças cardiovasculares e diabetes ${ }^{4}$. Apesar das doenças renais não terem associação com a mortalidade por covid-19 neste estudo, sabe-se que tais comorbidades pioram o prognóstico em pacientes com covid-19 e podem determinar o excedente de mortes, especialmente quando estão envolvidos diversos fatores ${ }^{21}$. O dano causado por condições metabólicas preexistentes como diabetes e hipertensão podem tornar os rins mais suscetíveis às lesões causadas pela infecção por covid-1922. Algumas condições de comprometimento imunológico podem contribuir para susceptibilidade às infecções por covid-19 e sua gravidade como câncer e pessoas infectadas com vírus da imunodeficiência humana (HIV) que apresentem contagens de linfócitos CD4 baixas ou carga viral não suprimida ${ }^{23}$.

As doenças respiratórias estiveram fortemente associadas com o excesso de mortalidade entre os infectados com covid-19. Elas são fator de risco para gravidade da doença e mortalidade por covid-193. Um estudo demonstrou que o SARS-CoV-2 pode causar sérios danos aos vasos sanguíneos e, consequentemente, induzir à trombose generalizada. Comparado à gripe, os pulmões de pessoas com covid-19 apresentaram nove vezes mais coágulos sanguíneos ${ }^{24}$. Portanto, apesar de ser um vírus respiratório, ele pode causar doença vascular com trombose e embolia, além de um possível efeito diabetogênico ${ }^{25}$.

A tosse e a dispneia foram observadas em $65,7 \%$ e $5,7 \%$ dos pacientes com doença não grave em uma revisão sistemática da população geral, respectivamente ${ }^{26}$. Em outro estudo com crianças, a tosse esteve presente em $46 \%$ dos pacientes com covid-1927. Sobretudo, estudos mostraram que a tosse esteve associada aos casos graves de covid-191, assim como a dispneia ${ }^{28}$, sendo proximamente relacionada aos casos que evoluíram para óbito ${ }^{29}$. Além disso, demonstrou-se que a tosse dos pacientes com covid-19 apresentam características latentes distintas de outras infecções respiratórias ${ }^{30}$.

Por fim, este estudo buscou unir as abordagens frequentista e bayesiana para tornar mais confiável e credível o modelo regressivo final proposto, sendo complementares 
uma da outra. Apesar disso, a base de dados disponibilizada pelo boletim epidemiológico no Rio Grande do Norte não é construída de maneira sistemática e uniforme, dificultando as análises em termos computacionais, sem apresentar o dicionário de dados, além de não incorporar informações sociodemográficas importantes como aspectos de renda, educação e condições de moradia dos indivíduos que poderiam ser utilizadas no modelo regressivo. Por outro lado, por ser uma doença pouco conhecida, há muitos aspectos da covid-19 que precisam ser elucidados pela comunidade científica, demandando estudos em diversos campos de saberes e práticas.

\section{CONCLUSÕES}

Os idosos apresentaram excedente de mortalidade por covid-19 no Rio Grande do Norte. Além disso, homens e pessoas que apresentaram comorbidades como diabetes, doenças cardíacas, doenças respiratórias e imunossupressão, além de sintomas como tosse e dispneia, estiveram associados à maior mortalidade. Os estudos científicos precisam concentrar esforços na elucidação das causas que levam estes grupos de risco aos excedentes de óbitos por covid-19, enquanto as políticas públicas de saúde precisam considerar estes fatores de risco de mortalidade, sendo o foco a doença e os mecanismos para combatê-la.

\section{REFERÊNCIAS}

1. Guan W. et al. Clinical characteristics of coronavirus disease 2019 in China. N. Engl. J. Med.2020; 382:1708-1720.

2. Zhang R, Li Y, Zhang AL, Wang Y, Molin M J. Identifying airborne transmission as the dominant route for the spread of COVID-19. Proc. Natl. Acad. Sci. 2020; 117:202009637.

3. Rod JE, Oviedo-Trespalacios O, Cortes-Ramirez J. A brief-review of the risk factors for covid-19 severity. Rev. Saude Publica. 2020; 54:60.

7. ORGANIZAÇÃO MUNDIAL DE SAÚDE. Archived: WHO Timeline - COVID-19. (2020).

4. Feng Y. et al. COVID-19 with Different Severities: A Multicenter Study of Clinical Features. Am. J. Respir. Crit. Care Med. 2020; 201:1380-1388.

5. Bastos L. S. et al. COVID-19 and hospitalizations for SARI in Brazil: a comparison up to the 12th epidemiological week of 2020. Cad Saude Publica. 2020; 36:e00070120.

6. Pelicioni PHS, Lord S R. COVID-19 will severely impact older people's lives, and in many more ways than you think! Brazilian J of Physical Therapy. 2020; 24: 293-294.

8. Chavarria-Miró, G. et al. Sentinel surveillance of SARSCoV-2 in wastewater anticipates the occurrence of COVID-19 cases. medRxiv 2020. 
9. Souza CDF, Paiva JPS, Leal TC, Silva LF, Santos LG. Evolução espaçotemporal da letalidade por COVID-19 no Brasil, 2020. J. Bras. Pneumol. 46:e20200208.

10. BRASIL. MINISTÉRIO DASAÚDE. Painel de casos de doença pelo coronavírus 2019 (COVID-19) no Brasil pelo Ministério da Saúde.

11. Thomas AM, Hoeltgebaum HH, Mishra S, Whittake C, Schnekenberg RP, et al. Report 21 - Estimating COVID-19 cases and reproduction number in Brazil. Imperial College London. 2020

12. Secretaria do Estado da Saúde Pública (SESAP). Rio Grande do Norte. Informe epidemiológico coronavírus 2019 (COVID-19).

13. Marinelli NP. et al. Evolução de indicadores e capacidade de atendimento no início da epidemia de COVID-19 no Nordeste do Brasil, 2020. Epidemiol. e Serv. Saude Rev. do Sist. Unico Saude do Bras. 2020; 29:e2020226.

14. Griffith DM. et al. Men and COVID-19: A Biopsychosocial Approach to Understanding Sex Differences in Mortality and Recommendations for Practice and Policy Interventions. Prev. Chronic Dis. 2020; 17:200247.

15. Bialek $\mathrm{S}$, et al. Severe Outcomes Among Patients with Coronavirus Disease 2019 (COVID-19) — United States, February 12-March 16, 2020. MMWR. Morb. Mortal. Wkly. Rep. 2020; 69:343-346.

16. Kalache A, et al. Aging and inequalities: social protection policies for older adults resulting from the Covid-19 pandemic in Brazil. Rev. Bras. Geriatr. e Gerontol. 2020; 23.

17. Pan $\mathrm{D}$, et al. The impact of ethnicity on clinical outcomes in COVID-19: A systematic review. E Clinical Medicine. 2020; 23:100404.

18. Webb Hoope M, Nápoles M,A, Pérez-Stable EJ. COVID-19 and Racial/Ethnic Disparities. JAMA - J. Am. Med. Assoc. 2020; 323:2466-2467.

19. Quiñones AR. et al. Racial/ethnic differences in multimorbidity development and chronic disease accumu- lation for middle-aged adults. PLoS One. 2019; 14 : $1-13$.

20. Price-Haywood EG, Burton J, Fort, Seoane L. Hospitalization and Mortality among Black Patients and White Patients with Covid-19. N. Engl. J. Med. 2020; 382:2534-2543.

21. Oyelade T, Alqahtani J, Canciani G. Prognosis of COVID-19 in Patients with Liver and Kidney Diseases: An Early Systematic Review and Meta-Analysis. Trop. Med. Infect. Dis. 2020; 5.

22. Ayres JS. A metabolic handbook for the COVID-19 pandemic. Nat. Metab.2019; 2.

23. Peixoto RD, Lopes MSM. COVID-19 among cancer patients. What we know so far? Brazilian J. Oncol. 2019;16:19-21.

24. Ackermann M. et al. Pulmonary vascular endothelialitis, thrombosis, and angiogenesis in Covid-19. N. Engl. J. Med. 2020; 383:120-128.

25. Price LC, McCabe C, Garfield B, Wort SJ. Thrombosis and COVID-19 pneumonia: the clot thickens! Eur. Respir. J. 2020;;2001608.

26. Zhao X, Zhang B, Li P, et al. Incidence, clinical characteristics and prognostic factor of patients with COVID-19: a systematic review and meta-analysis. medRxiv preprint (2020).

27. Chang TH, Wu JL, Chang LY. Clinical characteristics and diagnostic challenges of pediatric COVID-19: A systematic review and meta-analysis. J. Formos. Med. Assoc. 2020; 119:982-989.

28. Yang J. et al. Prevalence of comorbidities and its effects in patients infected with SARS-CoV-2: a systematic review and meta-analysis. Int. J. Infect. Dis. 2020; 94:91-95.

29. Yang K. et al. Clinical characteristics, outcomes, and risk factors for mortality in patients with cancer and COVID-19 in Hubei, China: a multicentre, retrospective, cohort study. Lancet Oncol. 2020; 21:904-913. 
30. Imran A. et al. AI4COVID-19: Al enabled preliminary diagnosis for COVID-19 from cough samples via an app. Informatics Med. Unlocked. 2020. 100378.

\section{CORRESPONDÊNCIA}

André Luiz Barbosa de Lima

Av. Rodrigues Alves, 766 - Tirol,

Natal-RN, CEP 59086-320.

E-mail: andreveterinario@hotmail.com 